\title{
A perfusion bioreactor-based 3D model of the subarachnoid space based on a meningeal tissue construct
}

\author{
Albert Neutzner ${ }^{1,2}$, Laura Power ${ }^{1,3,4}$, Markus Dürrenberger ${ }^{5}$, Hendrik P. N. Scholl ${ }^{6,7}$, Peter Meyer ${ }^{6}$, \\ Hanspeter E. Killer ${ }^{8}$, David Wendt ${ }^{1,3,4^{*}}$ and Corina Kohler ${ }^{1,2^{*}}$
}

\begin{abstract}
Background: Altered flow of cerebrospinal fluid (CSF) within the subarachnoid space (SAS) is connected to brain, but also optic nerve degenerative diseases. To overcome the lack of suitable in vitro models that faithfully recapitulate the intricate three-dimensional architecture, complex cellular interactions, and fluid dynamics within the SAS, we have developed a perfusion bioreactor-based 3D in vitro model using primary human meningothelial cells (MECs) to generate meningeal tissue constructs. We ultimately employed this model to evaluate the impact of impaired CSF flow as evidenced during optic nerve compartment syndrome on the transcriptomic landscape of MECs.
\end{abstract}

Methods: Primary human meningothelial cells (phMECs) were seeded and cultured on collagen scaffolds in a perfusion bioreactor to generate engineered meningeal tissue constructs. Engineered constructs were compared to human SAS and assessed for specific cell-cell interaction markers as well as for extracellular matrix proteins found in human meninges. Using the established model, meningeal tissue constructs were exposed to physiological and pathophysiological flow conditions simulating the impaired CSF flow associated with optic nerve compartment syndrome and RNA sequencing was performed.

Results: Engineered constructs displayed similar microarchitecture compared to human SAS with regards to pore size, geometry as well as interconnectivity. They stained positively for specific cell-cell interaction markers indicative of a functional meningeal tissue, as well as extracellular matrix proteins found in human meninges. Analysis by RNA sequencing revealed altered expression of genes associated with extracellular matrix remodeling, endo-lysosomal processing, and mitochondrial energy metabolism under pathophysiological flow conditions.

Conclusions: Alterations of these biological processes may not only interfere with critical MEC functions impacting CSF and hence optic nerve homeostasis, but may likely alter SAS structure, thereby further impeding cerebrospinal fluid flow. Future studies based on the established 3D model will lead to new insights into the role of MECs in the pathogenesis of optic nerve but also brain degenerative diseases.

Keywords: Optic nerve, Subarachnoid space, Meningothelial cells, Bioreactor, Optic nerve compartment syndrome, Cerebrospinal fluid

\footnotetext{
*Correspondence: David.Wendt@usb.ch; Corina.Kohler@unibas.ch

${ }^{1}$ Department of Biomedicine, University Hospital Basel \& University Basel,

Hebelstr. 20, 4031 Basel, Switzerland

Full list of author information is available at the end of the article
} 


\section{Background}

The flow of CSF within the SAS is indispensable for maintaining brain, spinal cord but also optic nerve function. CSF not only provides important cushioning, but is also essential for central nervous system homeostasis, sustaining nutrient supply to neuronal-glial networks, mediating transportation of signaling molecules and removing toxic metabolites [1]. Changes in CSF pressure, flow dynamics and composition have been related to age-linked and neurodegenerative diseases [2-4].

The SAS imposes a unique environment to its cellular component, the meningothelial cells (MECs) which are a key constituent of the dura mater and the leptomeninges (arachnoid and pia mater). The outermost meningothelial layer of the arachnoid fulfills a crucial function, forming a tight junction barrier thereby separating the CSF-filled SAS from the circulatory system in the dura [5]. From this layer, MECs extend into the SAS covering the collagen-enforced trabeculae, pillars and septae which ultimately submerge into the pia. As part of the pia, MECs form a thin mono-layer that is connected by gap-junctions and is in direct proximity to the basement membrane [6].

Within this intricate environment MECs are being subjected and adapted to continuous CSF flow. Under physiological conditions, CSF flow is tightly regulated and is likely important for maintaining MEC morphological properties as well as their physiological role. MECs synthesize key extracellular matrix (ECM) proteins of the meninges such as various collagens, as well as fibronectin, laminin and tenascin, important for pia basal lamina integrity and thus for neuronal support and function [7]. MECs also play a central role in CSF conditioning by secreting immune-modulating mediators including cytokines and chemokines, as well as neurotrophic factors and retinoic acid, which have been shown to promote axonal regeneration and differentiation [8-11]. In addition, MECs have been implicated in the clearance of waste products from the SAS through phagocytosis which is important for maintenance of neuronal tissue homeostasis [9, 12]. Taken together, alterations in the microenvironment of MECs could not only have potentially deleterious effects on MECs, but ultimately as well on neuronal function and survival.

To date, research on MECs has been hampered by the lack of suitable in vitro models that are capable of recapitulating the intricate three-dimensional architecture, complex cell-cell and cell-matrix interactions, and fluid dynamics within the SAS. Therefore, in this work we aimed to generate a state-of-the-art perfusion bioreactorbased 3D model of the SAS, based on an in vitro engineered meningeal tissue construct.
A pathology that highlights the importance of continuous CSF flow is optic nerve compartment syndrome (ONCS). ONCS is characterized by stagnating CSF flow within the peri-optic nerve SAS and is linked to a variety of optic neuropathies, such as normal tension glaucoma, papilledema and visual impairment and intracranial pressure syndrome [13-15]. Using the established model system, we finally aimed to simulate conditions of optic nerve compartment syndrome to study the effects of pathophysiological flow conditions on MEC function. Performing RNA sequencing, gene ontology, as well as pathway/network analyses, we determined that under pathological flow conditions genes involved in major physiological MEC functions were differentially regulated as compared to physiological flow conditions, thereby providing novel clues for a role of these cells in the pathogenesis of ONCS.

\section{Methods}

Perfusion bioreactor-based in vitro model

of the subarachnoid space

Model establishment

A tissue engineering strategy was used to establish a $3 \mathrm{D}$ in vitro model of the subarachnoid space based on a meningeal tissue construct. To this end, primary human meningothelial cells (phMECs) of leptomeningeal origin (Sciencell; Cat.no. \#1400; USA) were seeded at passage $6 / 7$ and cultured in a porous collagen scaffold (ULTRAFOAM $^{\mathrm{TM}}$; Bard, USA) within a perfusion bioreactor (Cellec Biotek, Switzerland) (Fig. 1A1).

The perfusion bioreactor system was used to first perfuse a cell suspension $(1 \mathrm{E}+06 \mathrm{phMECs})$ directly through the pores of the $3 \mathrm{D}$ scaffold $(8 \mathrm{~mm}$ diameter $\times 2 \mathrm{~mm}$ height) at a superficial velocity of $1.0 \mathrm{~mm} / \mathrm{s}$, in order to seed cells uniformly throughout the scaffold volume. After $24 \mathrm{~h}$ of perfusion seeding, the flowrate was reduced, and phMECs were cultured under perfusion (superficial velocity of $0.3 \mathrm{~mm} / \mathrm{s}$ ) for an additional $72 \mathrm{~h}$ to engineer a meningeal tissue construct.

\section{Morphological characterization of meningeal tissue constructs}

To assess the microarchitectural similarity between the engineered meningeal tissue constructs and in vivo SAS, scanning electron microscopy of an empty collagen scaffold, a meningeal tissue construct as well as of the SAS of a human optic nerve section (mid-orbital) was performed. To this end, human optic nerve was removed postmortem from a healthy donor, within $7 \mathrm{~h}$ after death. Written informed consent was obtained as part of the agreement for autopsy. Both globe and optic nerve was fixed in $2 \%$ glutaraldehyde $(0.1 \mathrm{M}$ cacodylate buffer) for 1 week. After dehydration of optic nerve segment using 


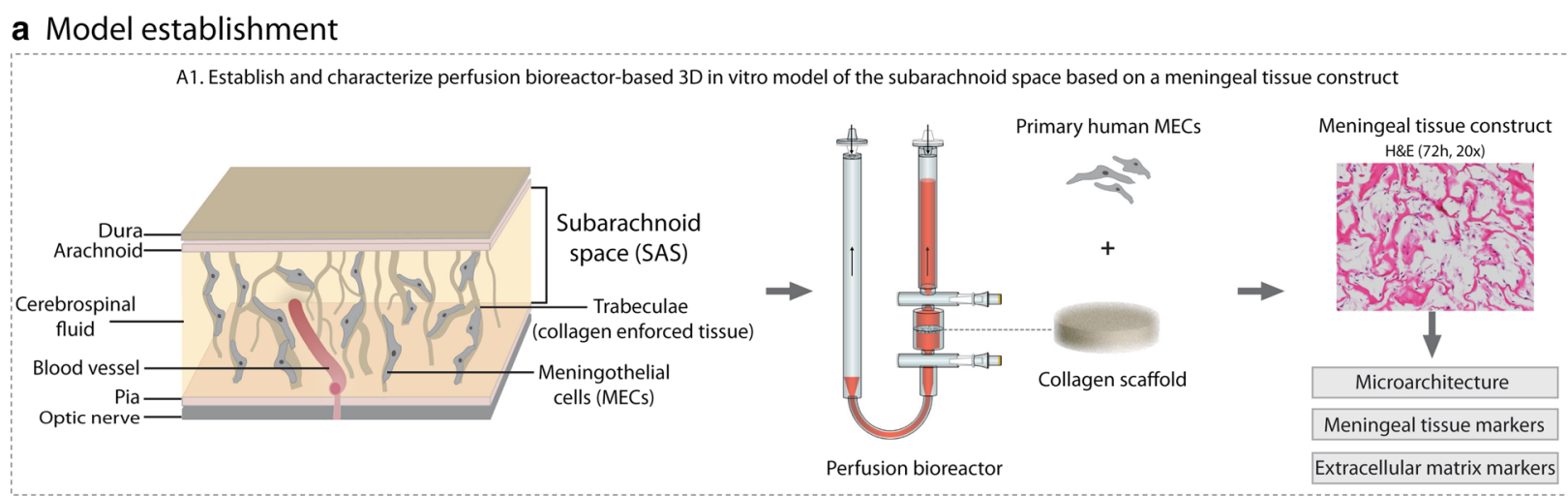

\section{b Model application}

B1. Evaluate oxygen supply to meningeal tissue constructs under different perfusion conditions

in vivo

oxygen supply via arterial system independent of CSF perfusion
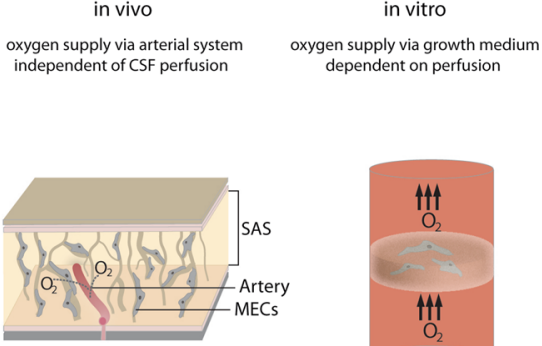
dependent on perfusion
den sula

measure $Q$ in bioreactor-based 3D model to ensure sufficient oxygen supply to MECs under pathophysiological perfusion
B2. Assess transcriptomic profile of primary human MECs under conditions of optic nerve compartment syndrome (pathophysiological perfusion) by RNA sequencing

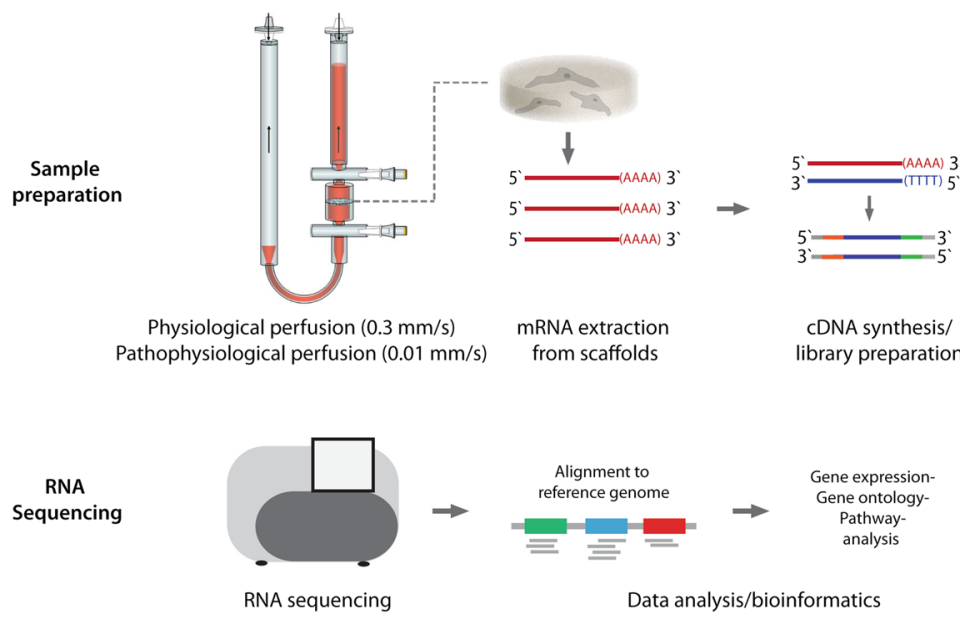

Fig. 1 Overview of model establishment and application. a Model establishment. To mimic SAS morphology and composition, U-CUP perfusion bioreactor was employed together with a collagen scaffold and phMECs were perfusion seeded at $1.0 \mathrm{~mm} / \mathrm{s}$ for $24 \mathrm{~h}$ and then cultivated for $72 \mathrm{~h}$ at a superficial velocity of $0.3 \mathrm{~mm} / \mathrm{s}$, to achieve a meningeal tissue construct. Tissue construct was characterized by assessing microarchitectural similarity between in vivo SAS and the engineered meningeal tissue construct employing scanning electron microscopy and by staining for meningeal tissue and extracellular matrix markers using IHC. b Model application. B1 To ensure sufficient oxygen supply to phMECs during pathophysiological perfusion, $\mathrm{O}_{2}$ saturation was measured during pathophysiological perfusion conditions. B2 Transcriptomic profile of phMECs was assessed during physiological and pathophysiological perfusion conditions of ONCS using RNA sequencing

an acetone series, critical point drying was performed. Samples were mounted onto aluminium holders and sputtered with gold $(15 \mathrm{~nm})$. Analyses was done on an SEM 505 (Philips, Einthoven, Netherlands). Meningeal tissue constructs were created as stated in the previous section. After $72 \mathrm{~h}$ of culturing constructs were removed from the bioreactor culture chamber, washed in TAM buffer for $10 \mathrm{~s}$, ultra rapid frozen in propane (according to Dubochet), transferred to liquid nitrogen and broken into pieces using a scalpel. This was followed by a $12 \mathrm{~h}$ freeze-drying program (stepwise increasing the temperature from 172 Kelvin to room temperature) using an EMS775X freeze drier (Quorum, UK). Samples were mounted onto aluminium holders and sputtered with gold $(20 \mathrm{~nm})$ at a vacuum of $2 \times 10^{-5}$ mbar. Empty scaffold and meningeal tissue construct were analyzed using a Nova Nano SEM 230 (FEI, NL).

Meningeal tissue constructs were characterized evaluating cell-cell as well as ECM protein expression, both characteristic features of meningeal tissue. Following $72 \mathrm{~h}$ of perfusion culture, engineered constructs were harvested, formalin fixed, paraffin embedded, and crosssectioned ( $4 \mu \mathrm{M}$ thick slices). To evaluate the cell distribution, hematoxylin and eosin (H\&E) staining was performed. For evaluation of cell-cell interaction markers scaffolds were stained using the following antibodies: 
Junctional adhesion molecule A, (Novus Biologicals, H00050848-M01), occludin (abcam, \#ab31721), claudin 5 (abcam, \#ab15106), connexin 43 (Sigma, \#C6219), connexin 26 (abcam, \#ab38584), desmoplakin I+ II (Progen, \#65146). For assessment of ECM markers, engineered constructs were stained using the following antibodies: Pro-collagen I (Fitzgerald, \#10R-1396), collagen II (MP BIOMEDICALS, \#0863171), IV (Ventana, \#7602632), laminin (Thermo, \#RB-082-A) and tenascin (FMI) (Fig. 1A1).

\section{Perfusion bioreactor-based in vitro model of ONCS Simulation of ONCS}

Using our bioreactor-based model of the SAS, phMECs were next cultured under pathophysiological perfusion conditions to simulate ONCS. phMEC-based constructs were engineered as described above. Flow rates were selected based on diffusion MRI measurements of flow-range ratios between the intracranial cavity and the subarachnoid space of the optic nerves (our unpublished observations). Regarding pathophysiological CSF flow occurring during ONCS, we used a flowrate in the bioreactor that was dramatically reduced to $2.5 \%$ of the normal flow, allowing for significantly inhibited flow while at the same time maintaining sufficient mass transport of oxygen to cells in the 3D construct, preventing hypoxic conditions. After $72 \mathrm{~h}$ of culture, flow rates for bioreactors simulating pathophysiological perfusion conditions were reduced to $0.01 \mathrm{~mm} / \mathrm{s}$ for $24 \mathrm{~h}(\mathrm{n}=6)$. As controls, engineered constructs were also maintained at physiological flow rates of $0.3 \mathrm{~mm} / \mathrm{s}$ for $24 \mathrm{~h}(\mathrm{n}=6)$ (Fig. 1B2). Oxygen measurements were performed to monitor oxygen concentrations supplied to phMECs within the 3D constructs under physiological $(0.3 \mathrm{~mm} / \mathrm{s})$ and pathophysiological $(0.01 \mathrm{~mm} / \mathrm{s})$ perfusion conditions. Therefore disposable flow-through chemo-optic micro-oxygen sensors (PreSens GmbH; Germany) were incorporated into the bioreactor system to obtain online measurements as previously described [16]. Measurements of oxygen levels were acquired every 10 min using Fibox 3 oxygen meters (PreSens GmbH; Germany). Two independent experiments with two bioreactors each were performed (Fig. 1B1).

\section{RNA extraction}

Engineered constructs were harvested in $1.5 \mathrm{ml}$ tubes, flash frozen on dry ice and RNA extraction was performed subsequently. To this end, frozen constructs were transferred into sterile petri dishes which were placed on dry ice. Constructs were cut into small pieces using a sterile scalpel, transferred into $1.5 \mathrm{ml}$ tubes, and cell lysis buffer added. After vortexing for $30 \mathrm{~s}$, centrifugation was performed at $2000 \mathrm{rpm}$ for $5 \mathrm{~min}$ at $4{ }^{\circ} \mathrm{C}$ and supernatant was transferred to new $1.5 \mathrm{ml}$ tubes. RNA extraction was performed using the Qiagen RNeasy Plus Mini Kit according to the manufacturer's protocol. To evaluate the quality of extracted RNA, samples were analyzed using Bioanalyzer Eukaryote Total RNA Nanochip (Agilent) (Fig. 1B2).

\section{Library generation and RNA sequencing}

Library preparation was performed using TruSeq Stranded mRNA Library Prep Kit (Illumina). In brief, poly-A containing mRNA molecules were purified using poly- $T$ oligo-attached magnetic beads. After purification, fragmentation of mRNA and first and second strand cDNA synthesis was performed. Following ligation of the adapter to the cDNA fragments, products were purified and PCR enriched to create the final cDNA library. The Libraries were sequenced on the NextSeq 500 system SR76 (Illumina). 26-46 million high quality reads were generated per library (Fig. 1B2).

\section{Bioinformatic analyses of RNA sequencing data}

Fastq files containing reads from MECs cultured under pathophysiological and physiological flow conditions were mapped to the human reference gene expression file (ftp://ftp.ensembl.org/pub/release89/fasta/homo_sapie ns/cdna/Homo_sapiens.GRCh38.cdna.all.fa.gz) using Salmon 0.8.2 [17]. To assess differential gene expression, a count matrix generated using R v3.3.1 and tximport v1.2.0 [18], was analyzed using DESeq2 v1.14.1 [19]. For analyzing targeted subsets of genes, as for evaluation of hypoxic response, GO terms for "hypoxia" were obtained from AmiGo [20, 21] and mapped to Ensembl ID using biomaRt [22]. Ingenuity Pathway Analysis (Qiagen) was used to identify biological and molecular networks involved in phMEC response under pathophysiological perfusion conditions.

\section{Results}

Perfusion bioreactor-based in vitro model

of the subarachnoid space

phMECs were perfusion seeded into porous 3D collagen scaffolds for $24 \mathrm{~h}$, then cultured for $72 \mathrm{~h}$. After $72 \mathrm{~h}$ of culture, phMECs were homogenously distributed throughout the collagen scaffold evident by $\mathrm{H} \& \mathrm{E}$ staining of constructs (Fig. 1A1). Microarchitectural similarity between empty collagen scaffold, engineered meningeal tissue construct and human SAS (optic nerve) was assessed by SEM. Pore sizes of collagen scaffold were in the range of $50-300 \mu \mathrm{M}$ which was comparable to the pore sizes observed in the meningeal tissue construct and optic nerve SAS. In addition empty collagen scaffolds possessed an interconnected pore structure which was 
as well present in engineered meningeal tissue constructs and in optic nerve SAS (Fig. 2).

Engineered meningeal tissue constructs were characterized by staining for cell-cell interaction markers as well as for ECM components, both characteristic hallmarks of native meningeal tissue. After 3 days, phMECs of the tissue-engineered meningeal constructs revealed strong immunopositivity for cell-cell interaction markers of gap junctions (connexin 26 and 43), and weak immunopositivity for desmosomes (desmoplakin) (Fig. 3a). In addition, engineered meningeal tissue constructs showed immunopositivity for ECM proteins pro-collagen, collagen type type II and type IV, laminin and tenascin (Fig. 3b).

\section{Perfusion bioreactor-based in vitro model of ONCS RNA sequencing}

To assess the response of phMECs under physiological and pathophysiological perfusion conditions with RNA sequencing, 6 engineered meningeal tissue constructs were generated under each perfusion condition. After RNA extraction from engineered constructs, Bioanalyzer Eukaryote Total RNA Nanochip (Agilent) was used to determine RNA concentration as well as RNA integrity. Digital electrophoresis revealed no degradation of RNA, evident by the presence of clear bands for $18 \mathrm{~S}$ as well as $28 \mathrm{~S}$ rRNA and RIN values ranging from 9.30 to 10.00 (Additional file 1: Figure S1). TruSeq Stranded mRNA Library Prep Kit yielded high quality libraries with library size distribution of an average of $346 \mathrm{bp}$. RNA sequencing generated 26-46 million high quality reads per library (Additional file 2: Figure S2).

\section{Gene expression patterns}

Following bioinformatics analysis using Salmon for transcript quantification and DESeq2 for differential gene expression analysis, 25,159 genes out of 38,837 genes were found to be expressed in phMECs. Out of these genes, 980 genes were found to be significantly, differentially regulated (threshold $=0$ ) between
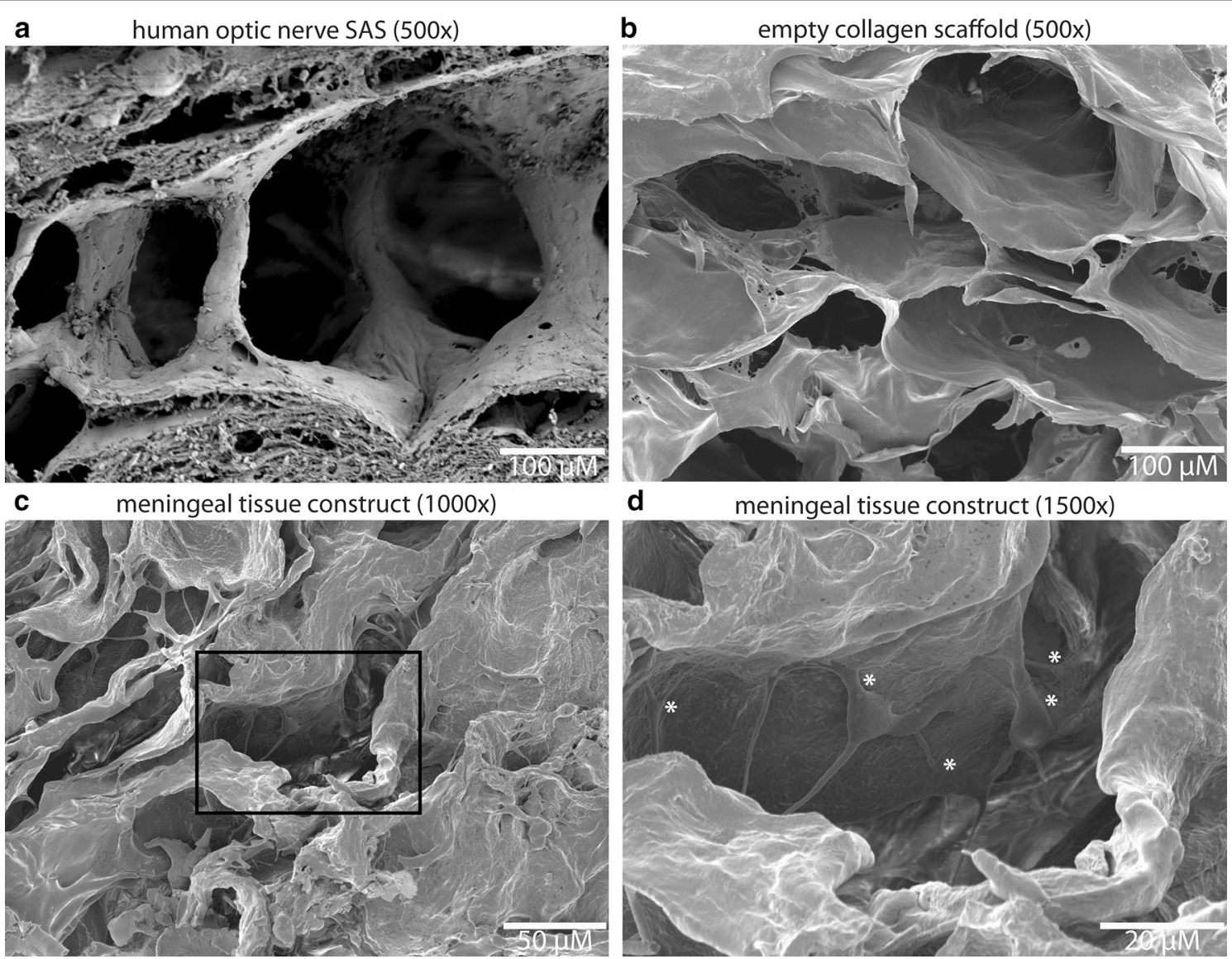

Fig. 2 Comparison of microarchitectural similarity between human optic nerve SAS, collagen scaffold and engineered meningeal tissue construct. SEM of human optic nerve SAS (a), empty collagen scaffold (b) and meningeal tissue construct (c, d) indicates similarity of the microarchitecture between the 3D in vitro model and in vivo SAS with regards to pore size, geometry and interconnectivity. ${ }^{*}$ Indicates meningothelial cells 


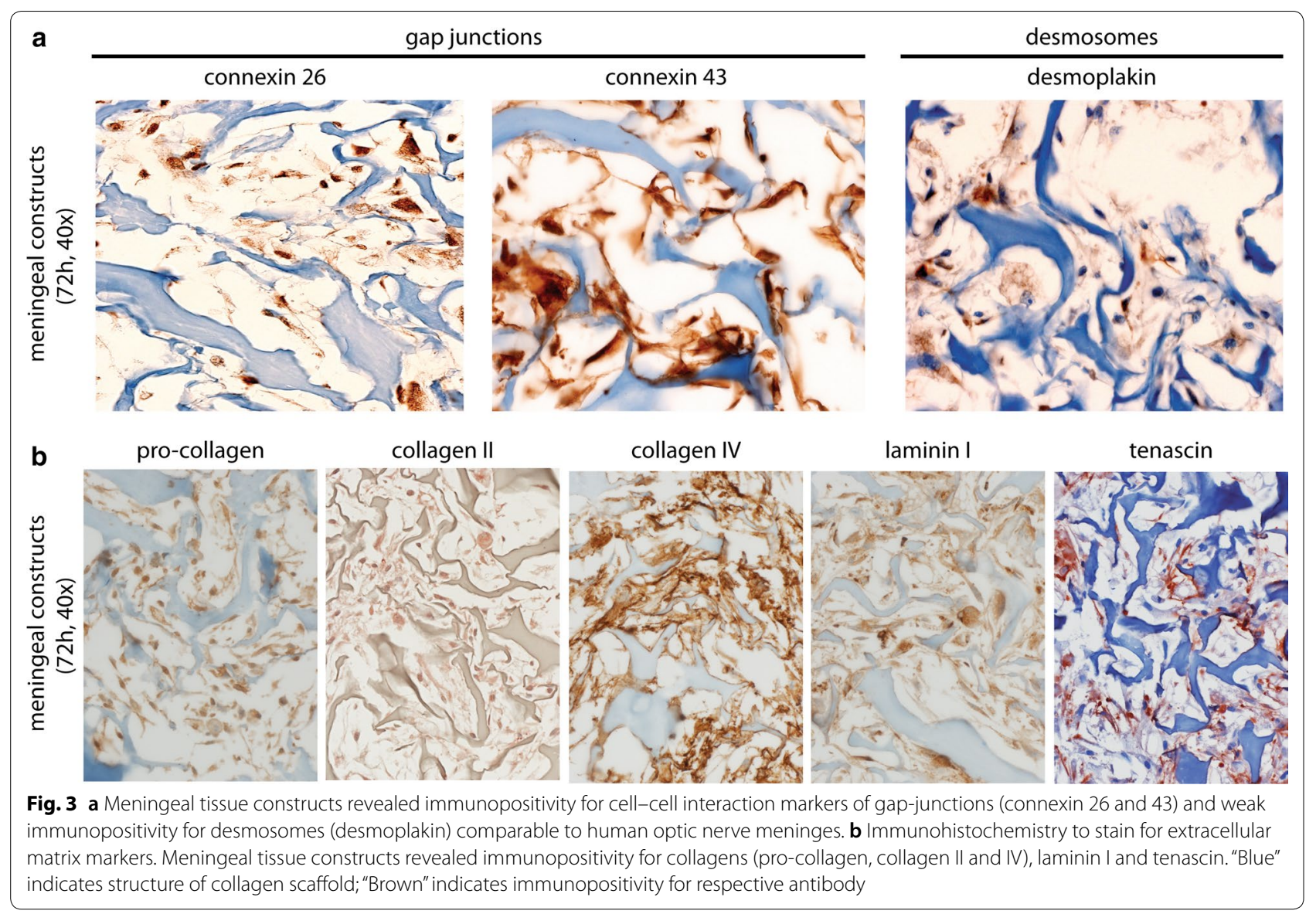

pathophysiological and physiological perfusion conditions (Fig. 4a). Applying a threshold of $0.5 \log 2$ foldchange, 96 genes were found to be significantly up- or down-regulated. Using principal component analysis (PCA), two main clusters corresponding to the treatment groups could be identified (Fig. 4b. These observations were confirmed in the sample distance analysis (Fig. 4c), with only one sample of the pathophysiological perfusion group clustering with the physiological perfusion group. An expression heatmap comparing Euclidian sample-tosample distance revealed clustering of pathophysiological and physiological perfusion samples. As shown in Fig. 4d, analysis of differentially regulated genes revealed strong clustering of the two treatment groups.

\section{Gene expression patterns of hypoxia related genes}

Within the optic nerve, oxygen is supplied to MECs by the vasculature. In our perfusion bioreactor-based model, since oxygen is supplied to cells within the engineered constructs by the perfusion of culture medium, we aimed to verify that the pathophysiological flow rate would not lead to hypoxic conditions in the engineered constructs, which could thereby influence gene expression and RNA sequencing results. Therefore, non-invasive micro-oxygen sensors were integrated into the bioreactor to monitor the level of oxygen supplied to MECs during culture. Figure 5a shows the average relative oxygen profile of two independent experiments with two biological replicates each. During $24 \mathrm{~h}$ of culture, only a minor drop in oxygen levels was observed. Oxygen levels in engineered constructs cultured under pathophysiological flow were maintained at $79.8 \pm 3.1 \%$ compared to levels measured at physiological flow. Based on these results, no hypoxic response would be expected under pathophysiological flow. We next aimed to corroborate the oxygen measurements and analyzed data from RNA sequencing for the expression pattern of 362 hypoxia related genes between physiological and pathophysiological perfusion conditions using hierarchical clustering. No significantly different gene expression patterns for hypoxia related genes between the two treatment groups were observed (Fig. 5b). Evaluation of significantly regulated hypoxia genes revealed both up-and downregulation of a small subset of genes, indicative for lack of specific hypoxic signaling (Fig. 5c). 


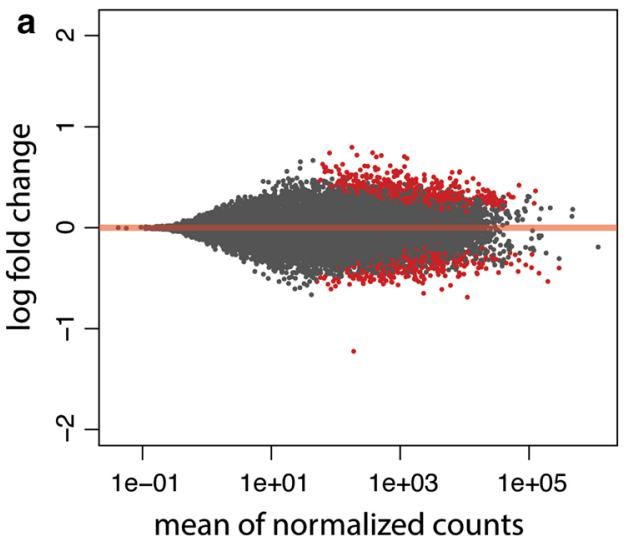

C

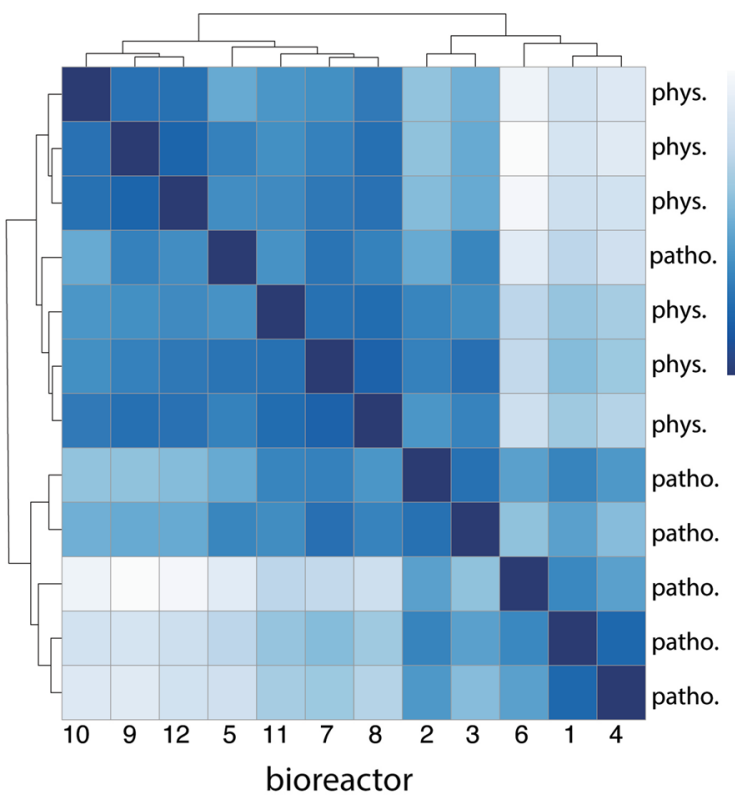

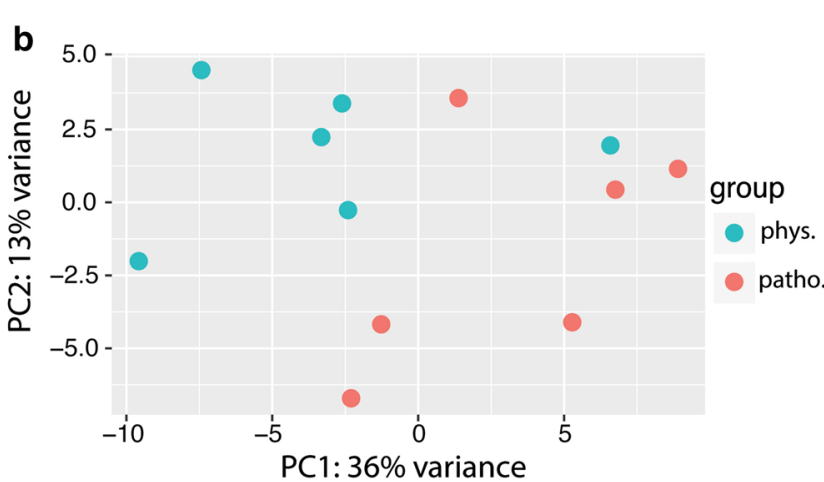

350000

$3 e+05$

250000

$2 \mathrm{e}+05$

150000

$1 e+05$

50000

50000

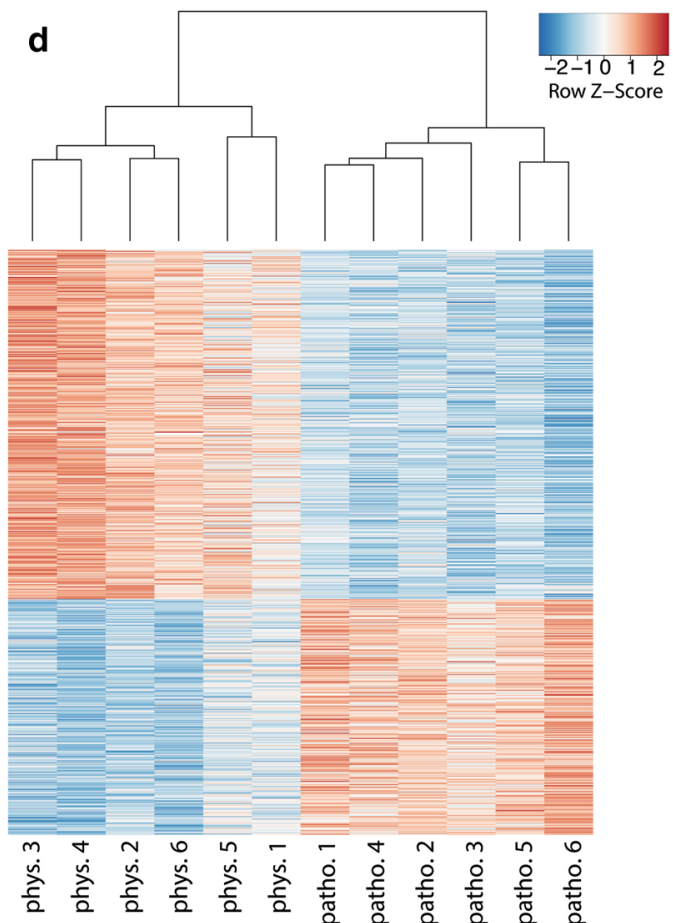

Fig. 4 Principal component analysis (PCA) and hierarchical clustering for comparison of gene expression patterns of phMECs under physiological (phys.) and pathophysiological (patho.) flow conditions. a MA plot showing DESeq2 filtered genes (25,159 genes). 980 of these genes were significantly regulated $(p<0.1$; shown in red). $\mathbf{b}$ PCA showing clustering of the two treatment groups, respectively. The fraction of the variance is 13\% for eigenvector 1 and 36\% for eigenvector 2. c Hierarchical cluster analysis of the two treatment groups recapitulating observations from PCA. d Expression heatmap showing Euclidian sample-to-sample distance matrix with hierarchical clustering of 980 differentially regulated genes in the 2 treatment groups

\section{Gene ontology (GO) analyses}

Enrichment analysis of gene ontology (GO) terms for the 980 differentially regulated genes revealed several processes, namely mRNA catabolism, cellular and mitochondrial bioenergetics, which were affected by pathophysiological flow (Additional file 3: Figure S3A). These processes were also identified when focusing on GO terms describing biological component (Additional file 3: Figure S3B) or biological function (Additional file 3: Figure S3C). Using REVIGO GO term refinement, significant differentially up-regulated genes were found in the categories of ECM organization, system development, peptide and protein metabolism and cell adhesion (Fig. 6a). Down-regulated genes were found in the categories of translation, signal recognition particle-dependent cotranslational protein targeting to membrane and cellular metabolic processes (Fig. 6b). 

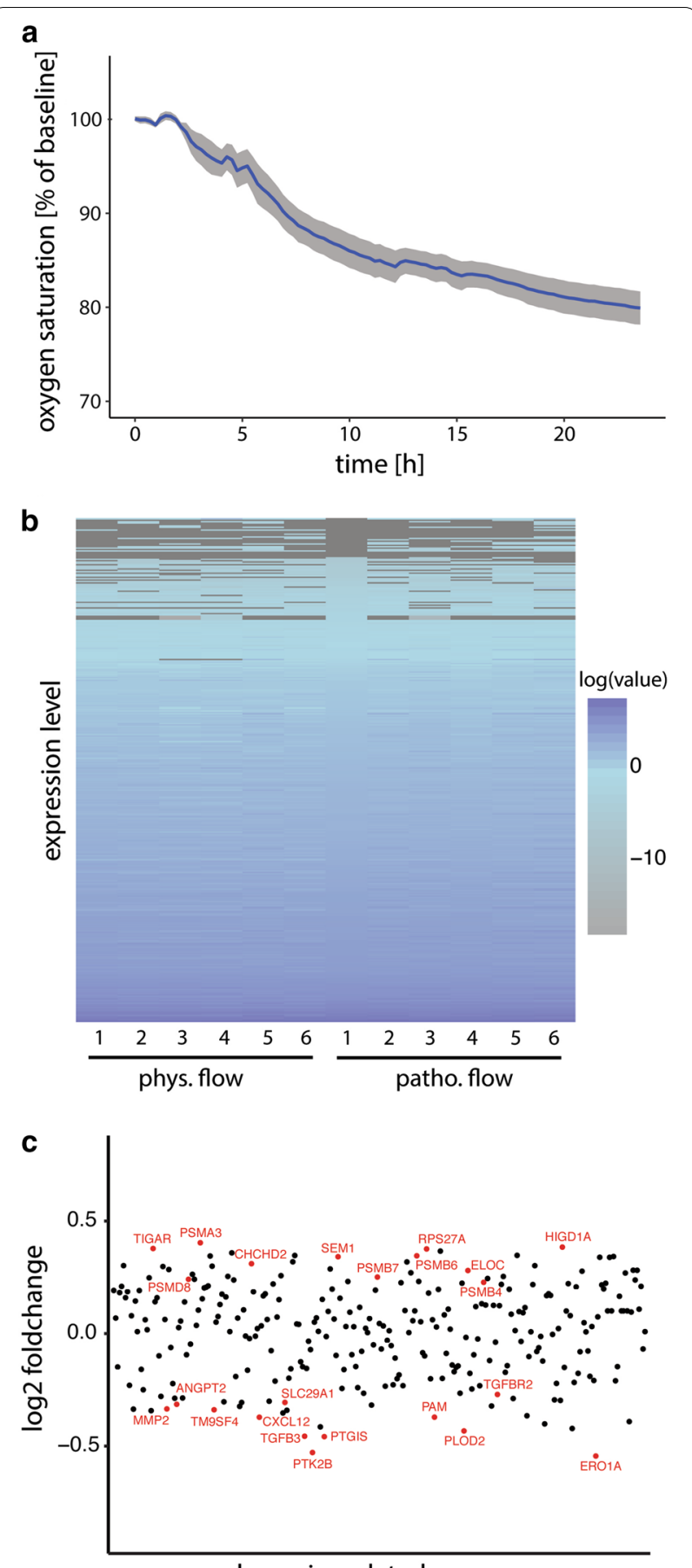

hypoxia-related genes

Fig. 5 Evaluation of oxygen supply to tissue engineered ONMs and comparison of expression of hypoxia-related genes under physiological (phys.) vs. pathophysiological (pathphys.) perfusion conditions. a Oxygen saturation following reduction of perfusion rate to $0.01 \mathrm{~mm} / \mathrm{s}$ ( $2.4 \%$ of physiological flow, relative to oxygen saturation under physiological flow). Shown is average of two independent experiments with two biological replicates each (S.D. in grey). b Expression heatmap showing Euclidian sample-to-sample distance matrix with hierarchical clustering of 362 hypoxia regulated genes in the 2 treatment groups. c Blot depicting significantly impacted hypoxia regulated genes

\section{Pathway analysis}

To gain further insight into the effect of pathophysiological flow on the regulation of biological networks in phMECs, pathway analysis using Ingenuity Pathway Analysis Software was performed. Consistent with our $\mathrm{GO}$ analysis, under pathophysiological flow, pathway analysis revealed an enrichment of up-regulated genes in processes including ECM organisation and endo-lysosomal processes. Genes upregulated in ECM organization included collagens (Type III, IV, XVIII) and laminin components (laminin, LAMB1, LAMA) but also integrins (integrin $\alpha$ ) (Fig. 7a). Within the endo-lysosomal pathway upregulated genes belonged to members of the protease (cathepsins $\mathrm{A}, \mathrm{L}$ and $\mathrm{F})$, galactosidase ( $\beta$-galactosidase) and mannosidase ( $\alpha$-mannosidase and $\beta$-mannosidase) families (Fig. 7b). An enrichment of down-regulated genes was found in the mitochondrial energy metabolic pathway. Within this pathway various genes coding for functional/structural components of the electron transport chain (Complex I, III, IV, V) were found to be downregulated (Fig. $7 \mathrm{c})$.

\section{Discussion}

We have established an in vitro 3D culture model as a surrogate for the SAS by culturing primary human MECs on collagen scaffolds within a perfusion bioreactor system. This model not only allows us to recapitulate the three-dimensional architecture, the complex cell-cell and cell-matrix interactions but also the fluid dynamics within this microenvironment, thereby making it a representative model to study MEC responses under physiological and pathophysiological conditions.

Studies applying computer assisted cisternography in patients with normal tension glaucoma and papilledema demonstrated impaired cerebrospinal fluid dynamics in the subarachnoid space of the optic nerve, a condition that is now described as optic nerve compartment syndrome (ONCS) [23-26]. Using our established model we demonstrated that pathophysiological flow conditions, as observed during ONCS, altered the trancriptomic profile of phMECs. Transcriptomic changes were observed in processes including extracellular matrix remodeling, endo-lysosomal processing and mitochondrial energy metabolism. We hypothesize that alterations of these processes might not only impact SAS architecture and, thus, flow dynamics but are also likely to interfere with major MEC functions, ultimately impacting optic nerve homeostasis.

The SAS possesses an intricate microarchitecture, being traversed by collagen reinforced trabeculae, pillars and septae which are covered with MECs [27]. Collagen scaffolds have been used for a number of tissue 


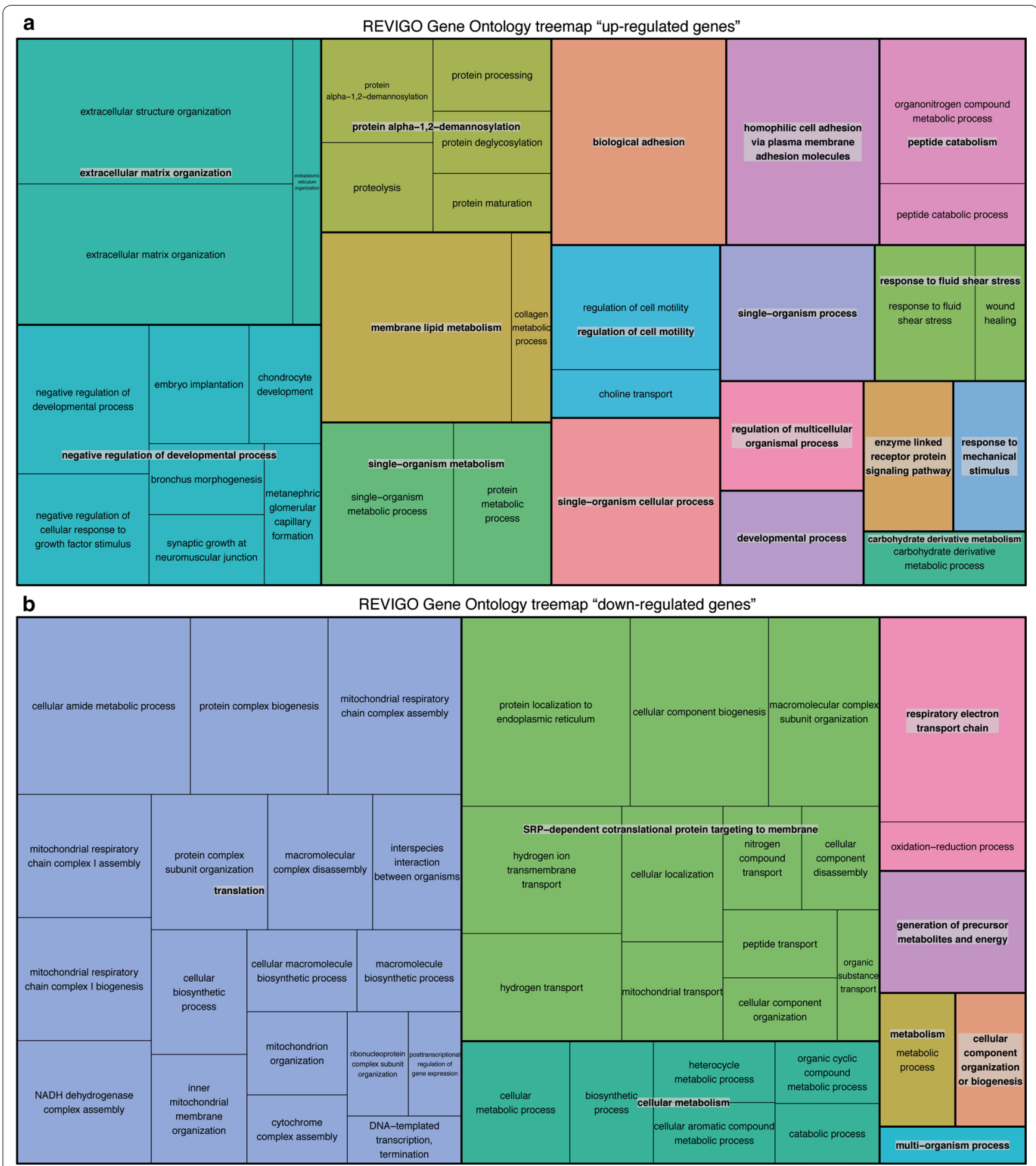

Fig. 6 REVIGO analyses of enriched GO-terms for up-regulated and down-regulated genes. a Up-regulated genes were found in the categories of extracellular matrix organization, negative regulation of developmental processes, peptide and protein metabolism and cell/biological adhesion. b Down-regulated genes were found in the categories of translation, SRP- dependent cotranslational protein targeting to membrane and cellular metabolic processes 
a

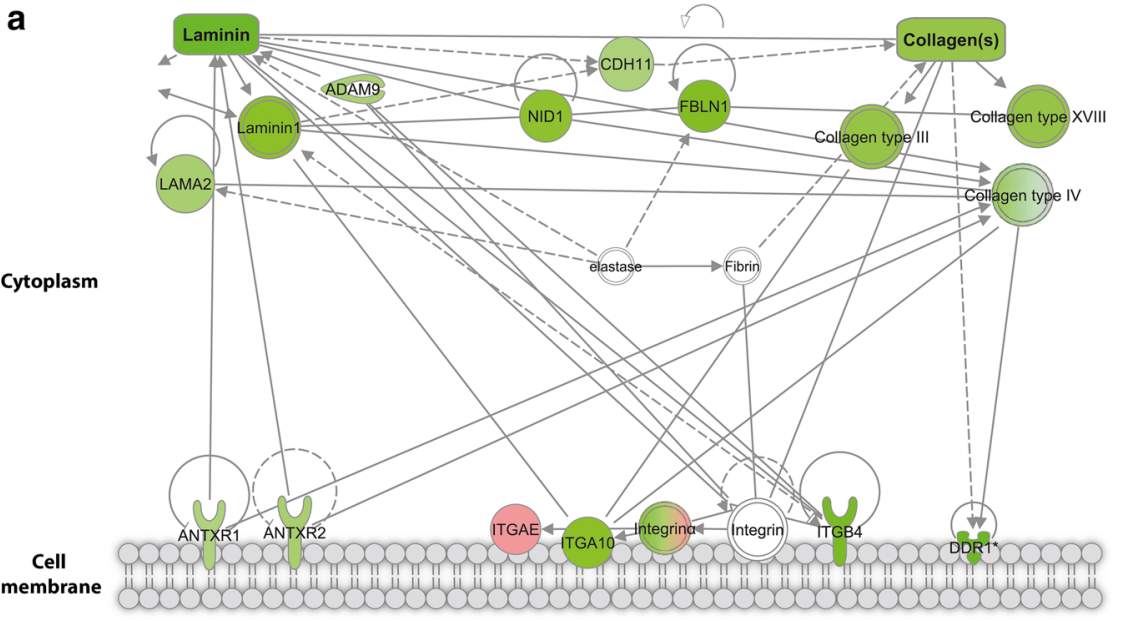

membrane

\begin{tabular}{|l|l|}
\hline - direct regulation & upregulated \\
--- indirect regulation & downregulated \\
\hline
\end{tabular}

b

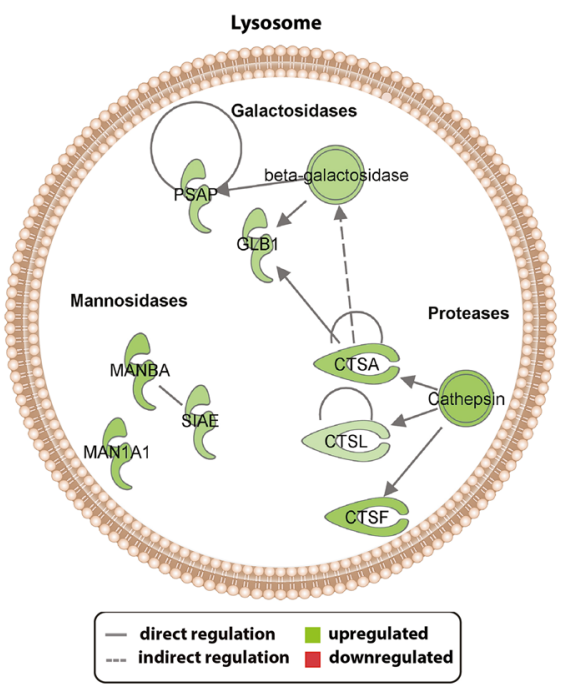

C

омM
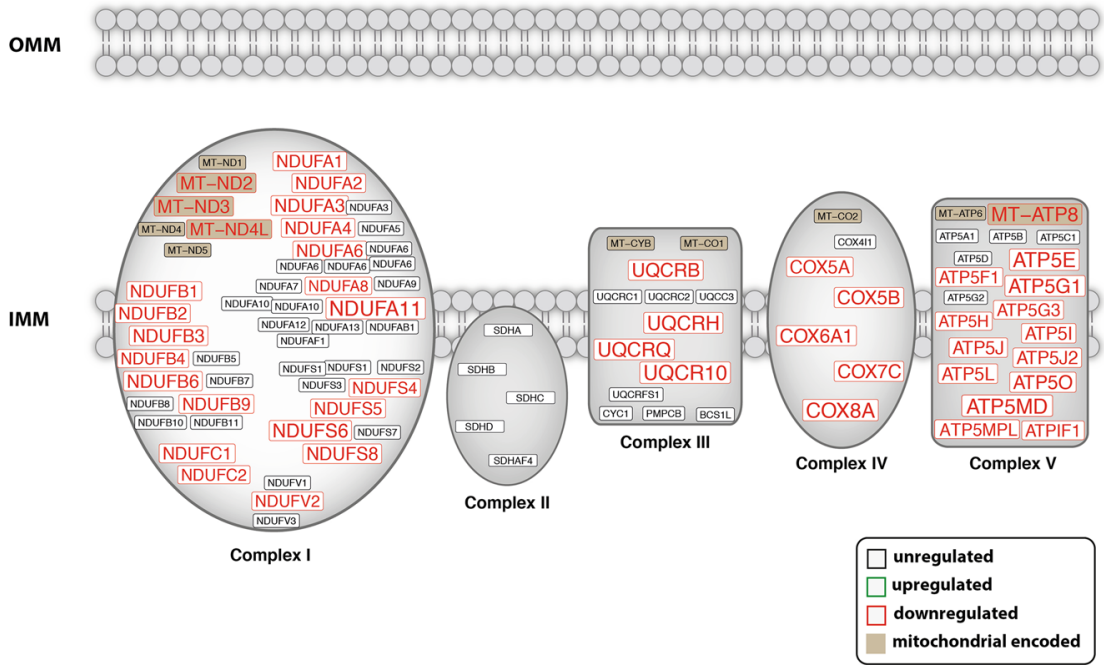
(See figure on previous page.)

Fig. 7 Ingenuity pathway analysis showing regulation of genes that code for components with functions in extracellular matrix remodeling, endo-lysosomal processing and mitochondrial energy metabolism. a For extracellular matrix remodeling up-regulated genes belong to members of the laminin (laminin 1/subunits a (LAMB1) and 32 (LAMA2)), collagen (Type III, XVIII, IV) and integrin family (Integrin a). b For endo-lysosomal processing up-regulated genes belonged to members of the protease (cathepsins $\mathrm{A}, \mathrm{L}$ and $\mathrm{F}$ ), galactosidase ( $\beta$-galactosidase) and mannosidase (a-mannosidase and $\beta$-mannosidase) families. c For mitochondrial energy metabolism down-regulated genes found were associated to ETC Complex I, III (ubiquinol binding proteins), IV (cytochrome C oxidase) and V (ATP-snthase) composition and function

engineering applications. Therefore, to mimic the SAS microarchitecture, biologically as well as structurally, we employed a collagen scaffold which has been shown to possess amorphous interconnected pores [28]. While porous collagen scaffolds have been used for numerous tissue engineering applications, for the first time, we have used a collagen scaffold for the in vitro engineering of meningeal tissue constructs. Scanning electron microscopy showed microarchitectural similarities between the empty collagen scaffold, the engineered meningeal tissue construct with the SAS of a human optic nerve (mid-orbital), with regards to pore size and geometry as well as interconnectivity. Within the SAS MECs form a tightly interconnected cellular network between the CSF and the CNS. While cell-cell interaction proteins are essential for maintaining barrier function and facilitating intercellular communication, meningeal-derived ECM components have been shown to be crucial for basal lamina integrity and thus for neuronal homeostasis. Thus, the presence of cell-cell interaction proteins and ECM components are characteristic features of a functional meningothelial tissue [9]. Characterization of the MECbased engineered constructs was therefore performed by staining for cell-cell interaction markers as well as ECM components. Immunohistochemistry of our MEC-based constructs demonstrated the presence of cell-cell interaction markers for gap-junctions and desmosomes that have previously been identified within human optic nerve meningeal tissues [29]. In addition, engineered meningeal tissue constructs were consistent with the composition of native tissues [7], staining immunopositive for the ECM components pro-collagen I, collagen II and IV as well as for laminin and tenascin.

Applying our model, we evaluated the response of MECs to conditions of pathophysiological flow dynamics as observed in ONCS and analyzed their transcriptional profile using RNA sequencing. Although there are studies of our and other groups that employ diffusion MRI to measure CSF fluid dynamics within the brain, spinal canal and optic nerve SAS [30,31], it is at the current time not feasible to obtain absolute CSF flow rates due to technical limitations. However it is possible to measure a so called flow-range ratio that allows the relative measurement of CSF flow rate by diffusion MRI imaging. Flow rates were selected based on diffusion MRI measurements of flow-range ratios between the intracranial cavity and the subarachnoid space of the optic nerves (our unpublished observations). To simulate the significantly reduced pathophysiological CSF flow occurring during ONCS, we used a flowrate in the bioreactor that was dramatically reduced to $2.5 \%$ of the normal flow. Although an absolute range of flowrates occurring in ONCS is still to be determined in the literature, the value selected for our model allowed for significantly inhibited flow while at the same time maintaining sufficient mass transport of oxygen to cells in the 3D construct, preventing hypoxic conditions.

RNA sequencing revealed a strong impact of pathophysiological flow on major physiologically relevant MEC functions. Although knowledge about the impact of fluid-induced shear stress on MECs is limited, previous work demonstrated that these cells are highly responsive to changes in fluid dynamics in vivo. Upon experimental blockage of CSF flow in sheep optic nerves, Jaggi et al. observed marked enlargement of MECs and of their intracellular structures [32]. Research on other cell types exposed to fluid-induced shear stress, such as vascular endothelial cells, provide additional evidence that altered fluid dynamics not only has major impact on cell phenotype but also on cell function. Changes in hemodynamics have been shown to initiate mechanosensitive signaling cascades in vascular endothelial cells resulting in modulation of gene expression and alteration of processes related to a variety of cellular functions including cytoskeletal remodeling [33], ECM homeostasis [34] and cellular metabolism [35]. These changes have major influence on vessel anatomy, integrity and function, making vascular endothelial cells a key player in physiological but also pathophysiological vascular remodeling $[36,37]$. It is hence conceivable that MECs might in a similar fashion respond to alterations in CSF dynamics.

Indeed, under pathophysiological flow conditions, RNA sequencing, GO-term analyses and REVIGO GOterm refinement revealed enrichment of up-regulated genes in categories related to changes in cellular, biochemical, and biomechanical properties such as ECM remodeling, biological adhesion and response to fluidinduced shear stress. We found upregulation of genes coding for collagens and laminin as well as for integrins. As MECs are known to secret key ECM components of 
the meninges, alterations in ECM homeostasis might have a severe influence on the microarchitecture of the optic nerve SAS. This might be particularly detrimental in individuals that present with a narrow optical canal. In this context, Wang et al. demonstrated a narrower orbital optic nerve subarachnoid space in normal tension glaucoma patients compared to controls and patients with primary open-angle glaucoma and elevated intraocular pressure [38]. In a study on 56 normal tension glaucoma patients Pircher et al. found a narrower optic canal compared to age-matched non glaucoma controls [39]. In such individuals MEC induced changes of subarachnoid space geometry might easier result in increased CSF flow resistance and finally in optic nerve compartmentation. In addition, as MECs secrete ECM components that contribute to the pial basal lamina, which is in direct contact with radial glial endfeet of the optic nerve fibers, altered ECM homeostasis by MECs would likely have an impact on the neuronal-glial network [40]. Indeed, there is evidence that alterations of ECM composition can lead to loss of basal membrane integrity, thereby interfering with neuronal-glial functioning. Mice that lack basal lamina components show defects in the attachment of radial glial cell processes at the meninges, which in turn has been shown to lead to enhanced neuronal progenitor apoptosis [41].

Disturbed shear stress has also been shown to induce an autophagic response and to modify bioenergetic processes thereby promoting cell survival [42-44]. Under stress conditions, autophagic clearance of damaged cellular organelles and proteins via the lysosomal pathway allow the cell to maintain cellular integrity. Increase of lysosomal components has thus been previously linked to autophagy induction. Wei et al. demonstrated that increased activation of Cathepsin $\mathrm{L}$ can stimulate autophagy and antagonize apoptosis in vascular endothelial cells thereby conferring an antiapoptotic effect [45]. In this regard, under pathophysiological perfusion conditions we observed upregulation of genes coding for components involved in endo-lysosomal processing including Proteases (Cathepsins A, L, F), Galactosidases and Mannosidases. It is reasonable to assume that in MECs the activation of an autophagic response might as well be a mechanism to cope with stress conditions induced by disturbed flow dynamics, thereby promoting cell survival. In addition, MECs have been implicated in the clearance of metabolic waste from the CSF and in maintaining CSF homeostasis $[9,10]$. As diminished CSF flow might lead to the accumulation of waste products we therefore hypothesize that reduced flow might be an "alarm signal" for MECs to adjust their degradation capacity to cope with an accumulation in waste products. Li et al. showed shear stress lead to the induction of oxidative stress which in turn mediated activation of Jun-N-terminal kinase, thereby initiating autophagy but as well inducing mitochondrial dysfunction due to impaired autophagic flux [35]. Interestingly, under pathophysiological flow conditions we also found down-regulation of genes involved in cellular metabolic processes and particularly in mitochondrial bioenergetics. Specifically, expression of genes involved in the electron transport chain complexes (I, III, IV and V) were impaired.

\section{Conclusion}

We report for the first time, a 3D in vitro model of the subarachnoid space based on an engineered meningeal tissue constructs. This novel bioreactor-based model recapitulates the three-dimensional architecture, complex cellular interactions, as well as fluid dynamics within the subarachnoid space. Applying this model allowed us to investigate fundamental aspects of MEC function under pathophysiological conditions simulating optic nerve compartment syndrome. Future studies based on this 3D model will allow to gain new insights into the role of MECs in the pathogenesis of optic nerve compartment syndrome and associated optic neuropathies and as well will be useful for the in vitro assessment of novel therapeutic treatments.

\section{Additional files}

Additional file 1: Figure S1. Agilent Bioanalyzer RNA quality assessment of physiological flow samples (1-6) and pathophysiological flow samples (6-12). A. Digital electrophoresis image depicting degradation profiles of RNA samples. All samples show clear bands for 185 and 285 rRNA indicative of high quality RNA. B. Electropherogram of each sample with corresponding RIN (RIN 9.3-10.0).

Additional file 2: Figure S2. TruSeq Stranded mRNA Library Prep quality assessment of physiological flow samples (1-6) and pathophysiological flow samples (6-12). A. Digital electrophoresis image depicting the library size distribution patterns, which reached on average $346.25 \mathrm{bp}$. B. Electropherogram of each sample showing library size distribution.

Additional file 3: Figure S3. Gene ontology (GO) analysis of RNA sequencing. $\mathrm{GO}$ analysis of 980 differentially regulated genes sorted according to Process (A), Component (B) and Function (C). q-value indicating GO-term enrichment.

\section{Abbreviations}

CSF: cerebrospinal fluid; ECM: extracellular matrix; MECs: meningothelial cells; ONCS: optic nerve compartment syndrome; phMECs: primary human meningothelial cells; SAS: subarachnoid space.

\section{Acknowledgements}

The authors want to acknowledge the D-BSSE genomics center, the Swiss Nanoscience Institute as well as DBM bioinformatics group for their support. The authors gratefully acknowledge Dr. Reza Asadollahi and Ms. Petra Hirschmann for their technical assistance.

\section{Authors' contributions}

AN together with LP and CK performed the main part of the practical laboratory work. MD established the protocol for the scanning electronmicroscopy 
analyses. AN and CK carried out the analyzes of the results. HPNS, PM and HEK made intellectual contributions. CK, DW and AN designed the study, CK and DW supervised the work and wrote the manuscript. All authors contributed to editing of the manuscript. All authors read and approved the final manuscript.

\section{Funding}

The authors are grateful for continued funding support provided by the Herta Messerli Foundation (Zurich, Switzerland) and by the Forschungsfonds Kantonsspital Aarau (Aarau, Switzerland).

\section{Availability of data and materials}

The datasets used and/or analysed during the current study are available from the corresponding author on reasonable request.

\section{Ethics approval and consent to participate}

Written informed consent was obtained as part of the agreement for autopsy.

\section{Consent for publication}

Not applicable.

\section{Competing interests}

The authors declare that they have no competing interests.

\section{Author details}

'Department of Biomedicine, University Hospital Basel \& University Basel, Hebelstr. 20, 4031 Basel, Switzerland. ${ }^{2}$ Department of Ophthalmology, University Hospital Basel \& University Basel, Hebelstr. 20, 4031 Basel, Switzerland. ${ }^{3}$ Department of Surgery, University Hospital Basel \& University Basel, Hebelstr. 20, 4031 Basel, Switzerland. ${ }^{4}$ Department of Biomedical Engineering, University Hospital Basel \& University Basel, Hebelstr. 20, 4031 Basel, Switzerland. ${ }^{5}$ Swiss Nanoscience Institute, University Basel, Klingelbergstr. 50, 4056 Basel, Switzerland. ${ }^{6}$ Department of Ophthalmology, University Hospital Basel \& University Basel, Mittlere Str. 91, 4056 Basel, Switzerland. ${ }^{7}$ Institute of Molecular and Clinical Ophthalmology, Mittlere Str. 91, 4056 Basel, Switzerland. ${ }^{8}$ Department of Ophthalmology, Kantonsspital Aarau, Tellstrasse 25, 5001 Aarau, Switzerland.

Received: 2 January 2019 Accepted: 22 May 2019 Published online: 13 June 2019

\section{References}

1. Whedon JM, Glassey D. Cerebrospinal fluid stasis and its clinical significance. Altern Ther Health Med. 2009;15(3):54-60.

2. May C, Kaye JA, Atack JR, Schapiro MB, Friedland RP, Rapoport SI. Cerebrospinal fluid production is reduced in healthy aging. Neurology. 1990;40(3 Pt 1):500-3.

3. Rubenstein E. Relationship of senescence of cerebrospinal fluid circulatory system to dementias of the aged. Lancet. 1998;351(9098):283-5.

4. Fishman RA. The cerebrospinal fluid production rate is reduced in dementia of the Alzheimer's type. Neurology. 2002;58(12):1866.

5. Nabeshima S, Reese TS, Landis DM, Brightman MW. Junctions in the meninges and marginal glia. J Comp Neurol. 1975;164(2):127-69.

6. Alcolado R, Weller RO, Parrish EP, Garrod D. The cranial arachnoid and pia mater in man: anatomical and ultrastructural observations. Neuropathol Appl Neurobiol. 1988;14(1):1-17.

7. Montagnani S, Castaldo C, Di Meglio F, Sciorio S, Giordano-Lanza G. Extra cellular matrix features in human meninges. Ital J Anat Embryol. 2000;105(3):167-77.

8. Li J, Fang L, Meyer P, Killer HE, Flammer J, Neutzner A. Anti-inflammatory response following uptake of apoptotic bodies by meningothelial cells. J Neuroinflamm. 2014;11:35.

9. Li J, Fang L, Killer HE, Flammer J, Meyer P, Neutzner A. Meningothelial cells as part of the central nervous system host defence. Biol Cell. 2013;105(7):304-15.

10. Fan B, Bordigari G, Flammer J, Killer HE, Meyer P, Neutzner A. Meningothelial cells participate in immunological processes in the cerebrospinal fluid. J Neuroimmunol. 2012;244(1-2):45-50.
11. Franzen R, Martin D, Daloze A, Moonen G, Schoenen J. Grafts of meningeal fibroblasts in adult rat spinal cord lesion promote axonal regrowth. Neuroreport. 1999;10(7):1551-6.

12. Zukor KA, Kent DT, Odelberg SJ. Meningeal cells and glia establish a permissive environment for axon regeneration after spinal cord injury in newts. Neural Dev. 2011;6:1.

13. Killer HE, Jaggi GP, Miller NR, Huber AR, Landolt H, Mironov A, et al. Cerebrospinal fluid dynamics between the basal cisterns and the subarachnoid space of the optic nerve in patients with papilloedema. Br J Ophthalmol. 2011;95(6):822-7.

14. Killer HE, Miller NR, Flammer J, Meyer P, Weinreb RN, Remonda L, et al. Cerebrospinal fluid exchange in the optic nerve in normal-tension glaucoma. Br J Ophthalmol. 2012;96(4):544-8.

15. Mader TH, Gibson CR, Pass AF, Lee AG, Killer HE, Hansen HC, et al. Optic disc edema in an astronaut after repeat long-duration space flight. $J$ Neuroophthalmol. 2013;33(3):249-55.

16. Wendt D, Stroebel S, Jakob M, John GT, Martin I. Uniform tissues engineered by seeding and culturing cells in 3D scaffolds under perfusion at defined oxygen tensions. Biorheology. 2006;43(3, 4):481-8.

17. Patro R, Duggal G, Love MI, Irizarry RA, Kingsford C. Salmon provides fast and bias-aware quantification of transcript expression. Nat Methods. 2017;14(4):417-9.

18. Soneson C, Love MI, Robinson MD. Differential analyses for RNA-seq: transcript-level estimates improve gene-level inferences. F1000Research. 2015:4:1521.

19. Love MI, Huber W, Anders S. Moderated estimation of fold change and dispersion for RNA-seq data with DESeq2. Genome Biol. 2014;15(12):550.

20. Ashburner M, Ball CA, Blake JA, Botstein D, Butler H, Cherry JM, et al. Gene ontology: tool for the unification of biology. The Gene Ontology Consortium. Nat Genet. 2000;25(1):25-9.

21. The Gene Ontology C. Expansion of the Gene Ontology knowledgebase and resources. Nucl Acids Res. 2017:45(D1):D331-8.

22. Smedley D, Haider S, Durinck S, Pandini L, Provero P, Allen J, et al. The BioMart community portal: an innovative alternative to large, centralized data repositories. Nucl Acids Res. 2015;43(W1):W589-98.

23. Pircher A, Montali M, Wostyn P, Pircher J, Berberat J, Remonda L, et al. Impaired cerebrospinal fluid dynamics along the entire optic nerve in normal-tension glaucoma. Acta Ophthalmol. 2018;96:e562-9.

24. Killer HE. Compartment syndromes of the optic nerve and open-angle glaucoma. J Glaucoma. 2013;22(Suppl 5):S19-20.

25. Killer HE, Subramanian PS. Compartmentalized cerebrospinal fluid. Int Ophthalmol Clin. 2014;54(1):95-102.

26. Boye D, Montali M, Miller NR, Pircher A, Gruber P, Killer HE, et al. Flow dynamics of cerebrospinal fluid between the intracranial cavity and the subarachnoid space of the optic nerve measured with a diffusion magnetic resonance imaging sequence in patients with normal tension glaucoma. Clin Exp Ophthalmol. 2018;46(5):511-8. https://doi. org/10.1111/ceo.13116.

27. Killer HE, Laeng HR, Flammer J, Groscurth P. Architecture of arachnoid trabeculae, pillars, and septa in the subarachnoid space of the human optic nerve: anatomy and clinical considerations. Br J Ophthalmol. 2003:87(6):777-81.

28. Seo YB, Lee OJ, Sultan MT, Lee JM, Park YR, Yeon YK, et al. In vitro and in vivo evaluation of the duck's feet collagen sponge for hemostatic applications. J Biomater Appl. 2017;32(4):484-91.

29. Zeleny TNC, Kohler C, Neutzner A, Killer HE, Meyer P. Cell-cell interaction proteins (gap junctions, tight junctions, and desmosomes) and water transporter aquaporin 4 in meningothelial cells of the human optic nerve. Front Neurol. 2017;8:308.

30. Dreha-Kulaczewski S, Konopka M, Joseph AA, Kollmeier J, Merboldt KD, Ludwig HC, et al. Respiration and the watershed of spinal CSF flow in humans. Sci Rep. 2018;8(1):5594.

31. Boye D, Montali M, Miller NR, Pircher A, Gruber P, Killer HE, et al. Flow dynamics of cerebrospinal fluid between the intracranial cavity and the subarachnoid space of the optic nerve measured with a diffusion magnetic resonance imaging sequence in patients with normal tension glaucoma. Clin Exp Ophthalmol. 2018;46(5):511-8.

32. Jaggi GP, Harlev M, Ziegler U, Dotan S, Miller NR, Killer HE. Cerebrospinal fluid segregation optic neuropathy: an experimental model and a hypothesis. Br J Ophthalmol. 2010;94(8):1088-93. 
33. Malek AM, Izumo S. Mechanism of endothelial cell shape change and cytoskeletal remodeling in response to fluid shear stress. J Cell Sci. 1996;109(Pt 4):713-26.

34. Humphrey JD, Dufresne ER, Schwartz MA. Mechanotransduction and extracellular matrix homeostasis. Nat Rev Mol Cell Biol. 2014;15(12):802-12.

35. Li R, Jen N, Wu L, Lee J, Fang K, Quigley K, et al. Disturbed flow induces autophagy, but impairs autophagic flux to perturb mitochondrial homeostasis. Antioxid Redox Signal. 2015:23(15):1207-19.

36. Collins C, Osborne LD, Guilluy C, Chen Z, O'Brien ET 3rd, Reader JS, et al. Haemodynamic and extracellular matrix cues regulate the mechanical phenotype and stiffness of aortic endothelial cells. Nat Commun. 2014;5:3984.

37. Diz-Munoz A, Fletcher DA, Weiner OD. Use the force: membrane tension as an organizer of cell shape and motility. Trends Cell Biol. 2013;23(2):47-53.

38. Wang N, Xie X, Yang D, Xian J, Li Y, Ren R, et al. Orbital cerebrospinal fluid space in glaucoma: the Beijing intracranial and intraocular pressure (iCOP) study. Ophthalmology. 2012;119(10):2065-2073.e1.

39. Pircher A, Montali M, Berberat J, Remonda L, Killer HE. The optic canal: a bottleneck for cerebrospinal fluid dynamics in normal-tension glaucoma? Front Neurol. 2017:8:47.

40. Sievers J, Pehlemann FW, Gude S, Berry M. Meningeal cells organize the superficial glia limitans of the cerebellum and produce components of both the interstitial matrix and the basement membrane. J Neurocytol. 1994;23(2):135-49.

41. Radakovits R, Barros CS, Belvindrah R, Patton B, Muller U. Regulation of radial glial survival by signals from the meninges. J Neurosci. 2009;29(24):7694-705.

42. Liu J, Bi X, Chen T, Zhang Q, Wang SX, Chiu JJ, et al. Shear stress regulates endothelial cell autophagy via redox regulation and Sirt1 expression. Cell Death Dis. 2015;6:e1827.

43. Yao $\mathrm{P}, \mathrm{Zhao} \mathrm{H}, \mathrm{MoW}, \mathrm{He}$ P. Laminar shear stress promotes vascular endothelial cell autophagy through upregulation with Rab4. DNA Cell Biol. 2016;35(3):118-23.

44. Breton-Romero R, Acin-Perez R, Rodriguez-Pascual F, Martinez-Molledo $M$, Brandes RP, Rial E, et al. Laminar shear stress regulates mitochondrial dynamics, bioenergetics responses and PRX3 activation in endothelial cells. Biochem Biophys Acta. 2014;1843(11):2403-13.

45. Wei DH, Jia XY, Liu YH, Guo FX, Tang ZH, Li XH, et al. Cathepsin L stimulates autophagy and inhibits apoptosis of ox-LDL-induced endothelial cells: potential role in atherosclerosis. Int J Mol Med. 2013;31(2):400-6.

\section{Publisher's Note}

Springer Nature remains neutral with regard to jurisdictional claims in published maps and institutional affiliations.
Ready to submit your research? Choose BMC and benefit from:

- fast, convenient online submission

- thorough peer review by experienced researchers in your field

- rapid publication on acceptance

- support for research data, including large and complex data types

- gold Open Access which fosters wider collaboration and increased citations

- maximum visibility for your research: over $100 \mathrm{M}$ website views per year

At $\mathrm{BMC}$, research is always in progress.

Learn more biomedcentral.com/submissions 